\title{
WIILEY
}

\section{Reference range determination for imaging biomarkers: Myocardial T1}

\begin{tabular}{|r|l|}
\hline Journal: & Journal of Magnetic Resonance Imaging \\
\hline Manuscript ID & JMRI-18-1394.R1 \\
\hline Wiley - Manuscript type: & Original Research \\
\hline Classification: & $\begin{array}{l}\text { Clinical Science, Cardiovascular and interventional imaging < Clinical } \\
\text { Science, Heart < Cardiovascular and interventional imaging }<\text { Clinical } \\
\text { Science }\end{array}$ \\
\hline Manuscript Keywords: & $\begin{array}{l}\text { Imaging biomarker, T1 mapping, reference range, reference interval, } \\
\text { normal values }\end{array}$ \\
\hline
\end{tabular}


Reference range determination for imaging biomarkers: Myocardial

T1 


\begin{abstract}
BACKGROUND: Imaging biomarkers, such as the T1 relaxation time of the myocardium using MRI, can be valuable in cardiac medicine if they are properly validated. Consensus statements recommend that for myocardial T1, each investigator should establish a reference range.
\end{abstract}

PURPOSE: To describe a statistically valid method for determining and reporting the reference range in each center, which simultaneously minimizes the twin risks of undersampling, leading to a uselessly uncertain range, and oversampling, which exposes volunteers to unnecessary scanning and wastes resources.

STUDY TYPE: Cohort

POPULATION: 278 normal human subjects without cardiac disease from two cardiac MR centers.

FIELD STRENGTH/SEQUENCE: 1.5T and 3T; Modified Look-Locker Inversion recovery sequence

ASSESSMENT: n/a The T1 relaxation time was estimated from multiple samples of tissue magnetization after inversion. A valid method for calculating a reference range is used.

STATISTICAL TESTS: Shapiro-Wilk test for Normality; Tukey robust approach for identification of outliers; reference range calculation with confidence intervals.

RESULTS: Reference ranges for measurement of myocardial T1 were calculated, with confidence intervals, enabling comparison with clinically important differences. At 3.0T: 1129 to $1301 \mathrm{~ms}$ at site $1(\mathrm{n}=21)$ and 1160 to $1309 \mathrm{~ms}$ at site $2(\mathrm{n}=59)$, and at $1.5 \mathrm{~T}$ at site 2 : 933 to $1020 \mathrm{~ms}$ (male, $\mathrm{n}=130$ ) and 965 to $1054 \mathrm{~ms}$ (female, $\mathrm{n}=68$ ). The $3 \mathrm{~T}$ reference range from site 1 was successfully benchmarked against the $3 \mathrm{~T}$ reference range at site 2 . 
DATA CONCLUSION: Myocardial T1 reference ranges can be properly characterized, enabling clinical comparison to a valid reference range with known confidence intervals, using methodology similar to that described in this report.

\section{Keywords}

Imaging biomarker, T1 mapping, reference range, reference interval, normal values 


\section{INTRODUCTION}

Imaging biomarkers play an increasing role in cardiac medicine. The BEST Resource (1) from the FDA-NIH Biomarker Working Group defines a biomarker as "a defined characteristic that is measured as an indicator of normal biological processes, pathogenic processes, or responses to an exposure or intervention, including therapeutic interventions". It continues “...molecular, histologic, radiographic, or physiologic characteristics are types of biomarkers", making imaging biomarkers such as the MR T1 relaxation time of the myocardium, if validated, formally equivalent to blood-borne molecular biomarkers such as troponin T and LDL-cholesterol, or to physiologic biomarkers such as systolic blood pressure.

A biomarker measured in similar patients with different equipment in different hospitals should yield similar values (2). This is an essential prerequisite before embarking on large multicenter outcome studies to create an evidence base for use. Unfortunately, for myocardial T1, this basic technical validation step is rather challenging. The design and operation of different manufacturers' MR equipment may affect accuracy, and in the case of myocardial fibrosis, even quite small differences in T1 may be biologically important. Recognising this risk of between-center irreproducibility, consensus statements recommend that each investigator should establish a reference range for myocardial $\mathrm{T} 1$ for his or her own equipment $(3,4)$.

A study to fully define the reference range involves a considerable number of subjects and significant costs - particularly if it has to be replicated by every investigator and for every new scanner installation or upgrade. More importantly it poses an ethical challenge: How best to define an adequate reference range while minimizing the exposure of volunteers to the burden of MR scanning from which they gain no benefit. 
The purpose of this work is to provide statistically valid methodology for investigators to calculate and interpret a reference range in their centre, while minimizing the twin risks of undersampling, leading to a uselessly uncertain range, and oversampling, which exposes volunteers to unnecessary scanning and wastes resources. Related tasks are also addressed, such as predicting the number of subjects needed, dealing with outliers, and benchmarking one reference range against another. Myocardial T1 reference ranges are reported. With the recent availability of mapping-based myocardial tissue characterization on clinical magnetic resonance systems, mapping of the $\mathrm{T} 1$ relaxation time is an exemplar imaging biomarker, but our approach is general.

\section{METHODS}

Data acquired for this work was acquired at site 1 using a healthy volunteer database (each subject provided informed consent and the study was approved by the local Ethics Review Board), and from site 2 using data from the local T1 quality control collective (aggregate data used for service evaluation does not require further ethical review (decision of the local Ethical Review Board)).

The calculation of four myocardial T1 reference ranges of subjects without cardiac disease is reported here: a $3 \mathrm{~T}$ reference range which includes both male and female subjects from site 1 $(\mathrm{n}=21,5$ male and 16 female, with age mean/sd 57/12y); a 3T reference range which includes both male and female subjects from site $2(n=59,38$ male and 21 female, with age mean/sd $52 / 9 \mathrm{y}) ; 1.5 \mathrm{~T}$ reference range for males at site $2(\mathrm{n}=132$ with age mean $/ \mathrm{sd} 50 / 8 \mathrm{y})$; and a $1.5 \mathrm{~T}$ reference range for females at site 2 ( $n=68$ with age mean/sd 48/10 y). Site 1 used a single Achieva TX MR system (Philips, Best, The Netherlands) using patient-adaptive RF shimming and a 6 channel receiver array. Site 2 used Ingenia MR systems (Philips, Best, The Netherlands) at both $1.5 \mathrm{~T}$ and 3.0T, both using a 28 channel receiver array. All acquisitions 
employed a MOLLI 5s(3s)3s product sequence (breath hold duration 11 seconds not dependent on heart rate). At 3.0T, sequence parameters include: acquired voxel size 2.0x2.0mm; TR/TE/flip angle $2.2 \mathrm{~ms} / 0.92 \mathrm{~ms} / 20^{\circ}$; SENSE acceleration factor 2, partial Fourier factors kx/ky 0.63/0.85; receiver bandwidth $1086 \mathrm{~Hz} / \mathrm{px}$; acquisition in end-systole (site 1) and end-diastole (site 2). At 1.5T, sequence parameters include: acquired voxel size 2.0x2.0mm; TR/TE/flip angle $2.4 \mathrm{~ms} / 1.1 \mathrm{~ms} / 35^{\circ}$; SENSE acceleration factor 2, partial Fourier factors $\mathrm{kx} / \mathrm{ky}$ 0.63/0.85; receiver bandwidth $1082 \mathrm{~Hz} / \mathrm{px}$; acquisition in end-diastole. No motion correction was applied and MOLLI source images were checked to ensure a steady breath hold position and myocardium alignment between source images. In cases of poor breath holding, the slice/map was reacquired. A blood correction was not applied. ROIs over the whole left ventricle myocardium were used at site 1 and over a left ventricle myocardium of a mid-slice at site 2 .

The Shapiro-Wilk test was employed to provide evidence that sample data are likely drawn from a Normally distributed population. Outliers are identified using the Tukey robust approach with box-and-whisker plots displaying interquartile ranges (IQR: Q3-Q1; Q1: lower quartile, Q3: upper quartile); at levels of $<$ Q1 - 1.5 IQR and / or $>$ Q3 + 1.5 IQR, the outliers are discarded $(5,6)$.

An ordered list of calculations which can be used to create a reference range is presented in Table 1. To facilitate the reader, various commands are identified for Microsoft Excel (2010 or later) and Google Sheets. Confidence intervals (CIs) are used to indicate a probability that values calculated from a random sample will include the population value of interest.

Calculated values are labelled in Table 1 and correspond to labels in Figure 1.

Thus, referring to Figure 1 and Table 1:

- the $95 \%$ reference range is from $\mathrm{J}$ to $\mathrm{H}$ 
- the $90 \%$ confidence intervals around the reference range limits are $\mathrm{R}$ to $\mathrm{Q}$, and $\mathrm{P}$ to $\mathrm{N}$

- the mean value is A with a $90 \%$ confidence interval of $\mathrm{U}$ to $\mathrm{T}$.

\section{RESULTS}

\section{Site 1 at 3.0T Males and Females}

Whole-heart ROI T1 data at 3 tesla for 21 normal subjects are listed here to allow repetition of calculations by the reader and were: $1245,1243,1299,1261,1243,1245,1144,1224$, $1133,1186,1179,1213,1202,1244,1220,1174,1212,1211,1157,1233,1245$ ms. Visual inspection of the data using a box-and-whisker plot confirmed no outliers are present (see Figure 2).

Using the Shapiro-Wilk test, the calculated W statistic was 0.96 , higher than the critical value of $\mathrm{W}$ of 0.91 (significance level 0.05), therefore the null hypothesis that the data are Normally distributed was not rejected. The data are likely consistent with the Normal distribution.

The $95 \%$ reference range is $1215 \pm 86 \mathrm{~ms}$. I.e. the $95 \%$ reference range is 1129 to $1301 \mathrm{~ms}$. The $90 \%$ CIs around the reference range limits are 1102 to $1156 \mathrm{~ms}$ and 1274 to $1328 \mathrm{~ms}$. These $90 \%$ CIs around the reference range limits each are $32 \%$ of the reference range size. The confidence intervals around the reference range limits allow us to state with increased confidence that a patient's T1 value is outside the reference range for the population, if it is outside the range from the lower CI boundary of the lower end of the reference range to the upper CI boundary of the upper end of the reference range (i.e. $\mathrm{R}$ to $\mathrm{N}$ in Figure 1). For this data set this corresponds to values less than 1102 and more than $1328 \mathrm{~ms}$.

The mean T1 in the normal subjects was $1215 \mathrm{~ms}$ with a $90 \%$ confidence interval of 1199 to $1230 \mathrm{~ms}$ (note that this is not the reference range). 


\section{Site 2 at 3.0T Males and Females}

The box-and-whisker plot confirmed no outliers are present (see Figure 2).

Using the Shapiro-Wilk test, the calculated W statistic was 0.99, higher than the critical value of W of 0.96 (significance level 0.05 ), therefore the null hypothesis that the data are Normally distributed was not rejected. The data are likely consistent with the Normal distribution.

The $95 \%$ reference range is $1234 \pm 75 \mathrm{~ms}$. I.e., the $95 \%$ reference range is 1160 to $1309 \mathrm{~ms}$. The $90 \%$ CIs around the reference range limits are 1145 to $1174 \mathrm{~ms}$ and 1295 to $1323 \mathrm{~ms}$. These $90 \%$ CIs around the reference range limits each are $19 \%$ of the reference range size. The range outside of which data may be more confidently placed outside the reference range for the population (i.e. $\mathrm{R}$ to $\mathrm{N}$ in Figure 1) for this data set this corresponds to 1145 to $1323 \mathrm{~ms}$.

The mean $\mathrm{T} 1$ in the normal subjects was $1234 \mathrm{~ms}$ with a $90 \%$ confidence interval of 1226 to $1242 \mathrm{~ms}$ (note that this is not the reference range).

\section{Site 2 at 1.5T Males}

The box-and-whisker plots showed two outliers present (see Figure 3). Upon clinical review of the outlier subjects, the high-T1 outlier was excluded due to clinical confounders. Using the Tukey robust approach described above it was confirmed that both outliers were outside the range for excluding unexplained outliers for this data $(920-1034 \mathrm{~ms})$, and therefore the low-T1 outlier was also omitted from the following results (i.e. updated $\mathrm{n}=130$ ).

Using the Shapiro-Wilk test, the calculated W statistic for the male T1 data is 0.99 , higher than the critical value of $\mathrm{W}$ of 0.98 (significance level 0.05). Therefore, the null hypothesis 
that the data are Normally distributed was not rejected. The data are likely consistent with the Normal distribution.

The $95 \%$ reference range is $976 \pm 44$ ms. I.e., the $95 \%$ reference range is 933 to $1020 \mathrm{~ms}$.

The $90 \%$ CIs around the reference range limits are 927 to $938 \mathrm{~ms}$ and 1014 to $1025 \mathrm{~ms}$. These $90 \%$ CIs around the reference range limits each are $13 \%$ of the reference range size. The range outside of which data may be more confidently placed outside the reference range for the population (i.e. $\mathrm{R}$ to $\mathrm{N}$ in Figure 1) for this data set this corresponds to 927 to $1025 \mathrm{~ms}$.

The mean T1 in the normal subjects was 976 ms with a $90 \%$ confidence interval of 973 to $979 \mathrm{~ms}$ (note this is not the reference range).

\section{Site 2 at 1.5T Females}

The box-and-whisker plot confirmed no outliers are present (see Figure 3).

Using the Shapiro-Wilk test, the calculated W statistic for female T1 data is 0.97 , higher than the critical value of $\mathrm{W}$ of 0.96 (significance level 0.05 ). Therefore the null hypothesis that the data are Normally distributed was not rejected. The data are likely consistent with the Normal distribution.

The $95 \%$ reference range is $1009 \pm 44 \mathrm{~ms}$. I.e., the $95 \%$ reference range is 965 to $1054 \mathrm{~ms}$.

The $90 \%$ CIs around the reference range limits are 957 to $973 \mathrm{~ms}$ and 1046 to $1061 \mathrm{~ms}$.

These $90 \%$ CIs around the reference range limits each are $18 \%$ of the reference range size.

The range outside of which data may be more confidently placed outside the reference range for the population (i.e. $\mathrm{R}$ to $\mathrm{N}$ in Figure 1) for this data set this corresponds to 957 to $1061 \mathrm{~ms}$. 
The mean T1 in the normal subjects was 1009 ms with a $90 \%$ confidence interval of 1005 to $1014 \mathrm{~ms}$ (note this is not the reference range).

Comparison of the reference ranges can be made with Table 2.

\section{DISCUSSION}

Thorough technical validation of an imaging biomarker includes assessment of whether the imaging biomarker measurement can be utilised in any geographical location and give comparable data (2). This requires an understanding of its repeatability (over measurements performed multiple times in the same subject using the same equipment, software and operators over a short timeframe), reproducibility (over measurements performed using different equipment, different software or operators, or at different sites and times, either in the same or in different subjects), and bias (systematic difference between measurements of a parameter and its real value). Even after minimizing all confounding technical and physiological pitfalls that influence the myocardial T1 (7), high quality reference ranges are still required to inform studies establishing clinical utility. The calculations and discussion provided in this work will enable clinical researchers using myocardial T1 measurement to create a robust local reference range and apply it carefully. It is not the intention of this work to review published data of myocardial T1 values; such reviews may be found in the literature (8). Nor is it proposed that the reference ranges reported in this work are ideal. Variations in workflow for any range may change utility, e.g., variations in pulse sequence used, whether motion correction is applied, how ROIs are placed including choice of segmentation, imaging in systole or diastole, subgroup analysis by gender or application of blood correction, et cetera. Rather, they illustrate that, whatever the end-to-end workflow of data acquisition and analysis, any reference range can be properly characterised and its utility more clearly understood, enabling appropriate use of a reference range in the clinical setting. 
The increased precision of the reference range limits can be appreciated with a larger number of subjects in our 3.0T data from two sites.

We use the term "reference range", outside of which T1 would be suspected to be abnormal, possibly affecting patient treatment. This term is equivalent to "reference interval"; we avoid the alternative term "normal range" as it may be confused with the Normal distribution. The reference population should be as similar as possible to the population on which the reference range will be applied. In some settings it may be necessary for a subgroup, such as older/younger age or male/female gender, to be considered with its own reference range; statistical criteria have been described for biochemical biomarkers which can identify such groups by focusing on the proportions of the subgroups outside the reference-range limits of the entire population (5). Whether subgroups are required will also depend on the effect size of the T1 change in disease. Subgroup reference ranges should not, however, be calculated unless there is a strong statistical rationale, as this increases the total required number of healthy participants, which must be counted separately in each subgroup.

For myocardial T1, data acquisition methods require attention. For example, in the case of Modified Look-Locker Imaging (MOLLI) T1 mapping methods as used in this work, particular attention should be paid to the MOLLI scheme; there are significant improvements in the performance of newer schemes as used in cardiac T1 mapping product software such as $5 s(3 s) 3 s$, versus the original MOLLI scheme $3 b(3 b) 3 b(3 b) 5 b(9)$. However, the discussions made in this work are not specific to MOLLI or to any specific MR system manufacturer; whatever T1 mapping method is used, the scanning procedures used to acquire the reference data should be performed in the same way when obtaining subsequent clinical data. Details such as the heart phase used for data acquisition may be noted; in normal myocardium, diastolic and systolic myocardial T1 values differ but correlate strongly, and blood correction reduces variability and gender differences $(10,11)$. 
Guidelines for recording region of interest (ROI) values from T1 maps are provided in the 2017 consensus statement (4). Where options are available, decisions should be recorded, so that the same approach can be mandated in subsequent diagnostic work, for example whether to draw an ROI on the septum only or over the whole myocardium. The mean pixel value of each ROI is recorded, which is used in reference range calculation. The standard deviation of individual ROIs would not generally be used in the calculation, but may also be recorded; if the standard deviations are very large or variable, it may be necessary review certain subjects' data.

The calculations which are described assume that the sample of healthy T1 values measured are drawn from a population which is Normally distributed. Though a Normal distribution of T1 values are expected, it is essential to check that the shape of the distribution of the sample data is consistent with a Normal distribution. It is incorrect to use the calculations below without preliminary verification or else an erroneous reference range might be created (5). Various parametric tests can be used to provide evidence that sample data are likely drawn from a Normally distributed population. The Shapiro-Wilk test is the most accurate test for small sample sizes and is recommended, although a range of methods are available (12). We are not aware of any previous findings of non-Normal distributions of healthy myocardial T1. In the unexpected scenario that sample data are not Normally distributed (or cannot be made Normal after transformation e.g. log, power, or some other function of the original scale), non-parametric statistics would be required, which are not covered here. Such a finding would have significant implications because although it simplifies calculation of the reference range, many more samples (healthy subjects) would be necessary.

Outliers may be discarded for a variety of reasons (conditions of the subject, measurement problems etc.). Nevertheless, sometimes outliers may occur with no such obvious reason for their occurrence. Whilst the Normal distribution allows for extreme values, such values may 
be removed if they are clinically implausible. Outliers can be identified from a visual inspection of the data, for example using the straightforward box-and-whisker plot using the ROI mean values (readily available in many spreadsheet applications and statistics packages/languages as well as online). In ambiguous cases a statistical method is useful to justify or rule out exclusion such as that described in this work.

A common reference range principle is to arbitrarily consider the central $95 \%$ of samples as being within the reference range, though adoption of a different interval may be acceptable with clinical justification. Here the standard $95 \%$ value is used.

The 2017 consensus statement (4) recommends calculation of the limits of the reference range "with the upper and lower range of normal defined by the mean plus and minus 2 standard deviations of the normal data". To contain $95 \%$ of all values in the population, the advised standard deviation multiplier of 2 is approximate, and valid only for large sample counts. The calculations described here provide a more accurate multiplier value using the Student's t-distribution which is appropriate when the sample size is small and the whole population (not sample) standard deviation is unknown, as is the case in this context. Careful reading of normative values reported in the literature is advised; the mean plus and minus only one standard deviation may be reported.

A reference range is intended to characterise a population. The correct interpretation of the reference range can have important consequences for patient care and should be done in consultation with a professional statistician. A distinction should be made between such descriptive statistics and clinical decision thresholds. A value within a reference range may be "typical" but it does not indicate "healthy". Similarly a value outside a reference range may be atypical but may not necessarily indicate disease. It is useful to remember that it is only an estimate of the true reference range which is, in general, unknowable, and hard 
thresholds for "normal" and "abnormal" reflect our choices in calculations and not nature.

Clinical decision thresholds are based on diagnostic questions, are defined by consensus, and are thresholds above or below which a specific medical decision is proposed (8). They are often derived from Receiver Operating Characteristic (ROC) curves and predictive values $(13,14)$. Where a reference range has wide confidence intervals around its reference range limits due to limited sample size, it may be prudent to use three classes of values instead of a standard normal/abnormal dichotomy, to manage the imprecision in the reference range limits (15). For example, the three classes of values may be: normal, indicating values within the reference range but more conservatively not including any values within the confidence intervals of the reference range limits (i.e. Q to $\mathrm{P}$, in Figure 1); indeterminate, indicating values contained within the confidence intervals of the reference range limits $(\mathrm{R}$ to $\mathrm{Q}$, and $\mathrm{P}$ to $\mathrm{N}$, in Figure 1); or abnormal, indicating values outside both the reference range and the confidence intervals of the reference range limits $(<\mathrm{R}$ and $>\mathrm{N}$ in Figure 1, see also extremes of 90\% CIs of reference range limits in Table 2). From a health-economic perspective, an "indeterminate" finding represents a futile use of MRI: if the proportion of "indeterminate" findings is unacceptably high this may suggest that the number of subjects used to determine the reference range was too low.

The number of healthy subjects, or normal individuals (referred to MR without any abnormal findings) required to create a reference range is not straightforward (16); it depends on the precision desired, which in turn depends on the proposed clinical application and diseases which may be encountered. Moreover, the number required also depends on the precision of the measurement method which varies from one method to the next (which for myocardial T1 mapping includes variations such as patient setup, system type, sequence used, analysis steps, etc). However some help can be given by asking a different question: How many samples are needed such that the confidence intervals for the reference range limits are at most a 
particular proportion ( $p$, e.g. one-third) of the reference range size? In other words, a judgement can be made about the precision of the limits of the reference range, compared to the width of the reference range itself; this can be visualised with the help of Figure 1 looking at $\mathrm{R}$ to $\mathrm{Q}$ (or $\mathrm{P}$ to $\mathrm{N}$ ) versus the reference range. We may estimate the number of samples needed by observing the following ( $c f 18 \mathrm{E} .1$. in ref. 16). The standard error of the limit of the reference range $\left(S E_{\text {lim }}\right.$, result $\mathrm{K}$ in Table 1$)$ may be approximated to $\sqrt{3 s^{2} / n}$ where $n$ is the number of samples and $s$ is the standard deviation. The confidence interval around the reference range limit is $2 t$ times $S E_{\text {lim }}$ where $t$ is the critical $\mathrm{t}$ value (result $\mathrm{L}$ in Table 1 ), and the width of the reference range is $2 t s$. Therefore, the proportion $p$ which we wish to calculate is $2 t S E_{\text {lim }} / 2 t s$, which reduces to $p=\sqrt{(3 / n)}$. Thus, the number of samples required to yield an acceptable proportion of the reference range size for the confidence intervals of the reference range limits is $n=3 / p^{2}$. Then, for example: if the acceptable proportion of the reference range size for the confidence intervals of the reference range limits is one quarter, the approximate number of samples required can be calculated using $3 /\left(0.25^{\wedge} 2\right)=48$

The number of subjects required for the reference range can be related to the expected $\mathrm{T} 1$ of myocardium for specific diseases, if the width of the reference interval is known or can be approximately established. Similarly, the utility of an existing reference range can be checked. For example: if a local myocardial T1 reference range at $1.5 \mathrm{~T}$ happens to be 940 to $1060 \mathrm{~ms}$, and in disease a hypothetical T1 of $920 \mathrm{~ms}$ is expected, in order to limit the confidence intervals of the reference interval limits to $40 \mathrm{~ms}( \pm(940-920))$ so that a value of $920 \mathrm{~ms}$ may be confidently interpreted as abnormal, the number of samples required can be calculated using $3 /\left((40 /(1060-940))^{\wedge} 2\right)=27$. 
For diseases in which the change in myocardial T1 is small (e.g. diseases causing diffuse myocardial fibrosis), smaller CIs around the reference range limits are required, necessitating a larger sample size when creating the reference range to be able to constructively classify patients' T1 values.

There are challenges involved in harmonising reference ranges from different institutions or from different equipment manufacturers. However, benchmarking local data against published reference ranges is possible and recommended (4). The following method is standardised practice in biochemistry (17). Twenty subjects' data (after removing outliers) are collected representing the local apparently healthy population satisfying the population criteria. Then, if no more than two of the 20 subjects fall outside the reference range, the reference range can be adopted, at least provisionally (5). If three or more are outside the reference range, the experiment should be repeated with another 20 subjects (after removing outliers). Again, if no more than two of the 20 subjects fall outside the reference range, the reference range can be adopted. If three or more are outside the reference range, the reference range is probably not valid and a local reference range should be created. This benchmarking verification method is valid where the population from which the reference values are measured has a Normal distribution. It is statistically sound; the probability of rejecting the reference range when in fact at least $95 \%$ of the whole local population do fall within the reference range being tested is 5 to $7 \%$ (17). In our 3.0T data from site 1 , it can be observed only two data points fall outside of the $3.0 \mathrm{~T}$ reference range from site 2 , despite differences in: specific scanner type; heart phase of data acquisition (end systole vs end diastole, respectively); and ROI inclusion criteria (whole heart vs only mid-slice, respectively). Thus, the benchmarking of the 3.0T reference ranges from site 1 against site 2 is successful. Reproducibility data on myocardial T1 measurement is accumulating in the literature, including: assessment in Fabry disease (18); inter-study, inter-observer and inter-center 
reproducibility in hemodialysis patients (19); at different field strengths (20); evaluation of different T1 mapping methods (21). It is advised that regularly repeated phantom-based quality control is performed to periodically confirm that the status and stability of the MR system have not changed significantly during the time between establishing a reference range and clinical scanning (4). Detailed instruction on quality control in MRI including of relaxometry, as well as discussion on appropriate phantom design, is available $(22,23)$.

A limitation of this study is the arbitrary numbers of normal subjects included from sites 1 and 2 , according to the data available at the time. These numbers are not designed to produce a reference range with a specific utility according to a particular clinical question.

Nevertheless, the reference ranges created show the steps to follow and considerations to make, and may be used for benchmarking.

In order for imaging biomarkers to cross clinical translation gaps, validation of the imaging biomarker must be robust. Myocardial T1 mapping in routine clinical practice still faces challenges including the creation of a local reference range of values, or the validation of a previously established and/or external reference range at the local site. The calculations and discussions provided in this work will equip the reader to perform these tasks. 
Acknowledgements: We thank the Cardiovascular Medicine Research team at the University of Aberdeen for acquisition of healthy volunteer data. 


\section{References}

1. FDA-NIH Biomarker Working Group. BEST (Biomarkers, EndpointS, and other Tools) Resource [Internet]. Silver Spring (MD): Food and Drug Administration (US); 2016-. Glossary. 2016 Jan 28 [Updated 2018 May 2]. https://www.ncbi.nlm.nih.gov/books/NBK338448/ accessed 28th September 2018

2. O'Connor JP, Aboagye EO, Adams JE, et al. Imaging biomarker roadmap for cancer studies. Nat Rev Clin Oncol. 2017;14:169-186.

3. Moon JC, Messroghli DR, Kellman P, et al. Myocardial T1 mapping and extracellular volume quantification: a Society for Cardiovascular Magnetic Resonance (SCMR) and CMR Working Group of the European Society of Cardiology consensus statement. J Cardiovasc Magn Reson. 2013;15:92.

4. Messroghli DR, Moon JC, Ferreira VM, et al. Clinical recommendations for cardiovascular magnetic resonance mapping of T1, T2, T2* and extracellular volume: A consensus statement by the Society for Cardiovascular Magnetic Resonance (SCMR) endorsed by the European Association for Cardiovascular Imaging (EACVI). J Cardiovasc Magn Reson. $2017 ; 19: 75$.

5. Ceriotti F, Hinzmann R, Panteghini M. Reference intervals: the way forward. Ann Clin Biochem. 2009;46:8-17.

6. Ozarda Y. Reference intervals: Current status, recent developments and future considerations. Biochemia Medica. 2016;26:5-11.

7. Cameron D, Vassiliou VS, Higgins DM, Gatehouse PD. Towards accurate and precise T1 and extracellular volume mapping in the myocardium: a guide to current pitfalls and their solutions. Magn Reson Mater Phy 2018;31:143-163. 
8. Puntmann VO, Peker E, Chandrashekhar Y, Nagel E. T1 Mapping in Characterizing Myocardial Disease: A Comprehensive Review. Circ Res. 2016;119:277-99.

9. Higgins DM and Moon JC. Review of T1 Mapping Methods: Comparative Effectiveness Including Reproducibility Issues. Curr Cardiovasc Imaging Rep 2014;7:9252.

10. Reiter U, Reiter G, Dorr K, Greiser A, Maderthaner R, Fuchsjäger M. Normal diastolic and systolic myocardial T1 values at 1.5-T MR imaging: correlations and blood normalization. Radiology. 2014;271:365-72.

11. Nickander J, Lundin M, Abdula G, et al. Blood correction reduces variability and gender differences in native myocardial $\mathrm{T} 1$ values at $1.5 \mathrm{~T}$ cardiovascular magnetic resonance - a derivation/validation approach. J Cardiovasc Magn Reson. 2017;19:41.

12. Ghasemi A, Zahediasl S. Normality Tests for Statistical Analysis: A Guide for NonStatisticians. Int J Endocrinol Metab 2012;10:486-489.

13. Sikaris KA. Physiology and its Importance for Reference Intervals. Clin Biochem Rev. 2014;35:3-14.

14. Sikaris K. Application of the Stockholm hierarchy to defining the quality of reference intervals and clinical decision limits. Clin Biochem Rev. 2012;33:141-8

15. Leslie WD, Greenberg ID. Reference range determination: the problem of small sample sizes. J Nucl Med. 1991;32:2306-10.

16. Bland, M. An Introduction to Medical Statistics. 3rd Ed. New York: Oxford University Press; 2001, 405 p.

17. CLSI document C28-A3c, 2008. CLSI. Defining, Establishing, and Verifying Reference Intervals in the Clinical Laboratory; Approved Guideline - Third Edition. Wayne, PA: Clinical Laboratory and Standards Institute. 
18. Pica S, Sado DM, Maestrini V, et al. Reproducibility of native myocardial T1 mapping in the assessment of Fabry disease and its role in early detection of cardiac involvement by cardiovascular magnetic resonance. J Cardiovasc Magn Reson. 2014;16:99.

19. Graham-Brown MP, Rutherford E, Levelt E, et al. Native T1 mapping: inter-study, interobserver and inter-center reproducibility in hemodialysis patients. J Cardiovasc Magn Reson. 2017;19:21.

20. Raman FS, Kawel-Boehm N, Gai N, et al. Modified look-locker inversion recovery T1 mapping indices: assessment of accuracy and reproducibility between magnetic resonance scanners. J Cardiovasc Magn Reson. 2013;15:64.

21. Roujol S, Weingärtner S, Foppa M, et al. Accuracy, precision, and reproducibility of four T1 mapping sequences: a head-to-head comparison of MOLLI, ShMOLLI, SASHA, and SAPPHIRE. Radiology. 2014;272:683-689.

22. Quality Control and Artefacts in Magnetic Resonance Imaging. Report 112, Institute of Physics and Engineering in Medicine (IPEM). 1st Ed. York: IPEM; 2017, 222 p.

23. Captur G, Gatehouse P, Keenan KE, et al. A medical device-grade T1 and ECV phantom for global T1 mapping quality assurance-the T1 Mapping and ECV Standardization in cardiovascular magnetic resonance (T1MES) program. J Cardiovasc Magn Reson. $2016 ; 18: 58$. 
Tables

\begin{tabular}{|c|c|c|c|}
\hline Value & Spreadsheet & Comment & Result label \\
\hline Mean & AVERAGE $(<$ range $>)$ & & A \\
\hline Standard deviation & STDEV.S $(<$ range $>)$ & Appropriate for a sample & B \\
\hline Sample size & COUNT $(<$ range $>)$ & & $\mathrm{C}$ \\
\hline Degrees of freedom & $\mathrm{C}-1$ & & $\mathrm{D}$ \\
\hline Significance level & $1-0.95$ & $\begin{array}{l}\text { One, minus proportion of } \\
\text { values to be considered } \\
\text { "normal", usually } 95 \%\end{array}$ & E \\
\hline $\begin{array}{l}\text { Critical t value for } \\
\text { reference interval }\end{array}$ & T.INV.2T(E,D) & Two-tailed & $\mathrm{F}$ \\
\hline $\begin{array}{l}\text { Reference interval } \\
\text { tolerance }\end{array}$ & $\mathrm{B} * \mathrm{~F}$ & $\begin{array}{l}\text { This result is one side of } \\
\text { the reference range }\end{array}$ & $\mathrm{G}$ \\
\hline $\begin{array}{l}\text { Upper limit of } 95 \% \\
\text { reference range }\end{array}$ & $A+G$ & & $\mathrm{H}$ \\
\hline $\begin{array}{l}\text { Lower limit of } 95 \% \\
\text { reference range }\end{array}$ & $A-G$ & & $\mathrm{~J}$ \\
\hline $\begin{array}{l}\text { Standard error of the } \\
\text { tolerance }\end{array}$ & $\mathrm{SQRT}\left(\mathrm{B}^{\wedge} 2 *((1 / \mathrm{C})+(2 /(\mathrm{D})))\right)$ & $\begin{array}{l}\text { Will be used for } \\
\text { confidence interval } \\
\text { around reference interval } \\
\text { limits }\end{array}$ & $\mathrm{K}$ \\
\hline $\begin{array}{l}\text { Critical t value for } \\
\text { reference interval limits }\end{array}$ & T.INV.2T(1-0.9,D) & Typically $90 \% \mathrm{CI}$ is used & $\mathrm{L}$ \\
\hline $\begin{array}{l}\text { Reference range limits } \\
\text { tolerance }\end{array}$ & $\mathrm{L}^{*} \mathrm{~K}$ & $\begin{array}{l}\text { This result is one side of } \\
\text { the range-limit CI }\end{array}$ & M \\
\hline $\begin{array}{l}\text { Upper } 90 \% \text { CI limit for } \\
\text { upper limit of reference } \\
\text { range }\end{array}$ & $\mathrm{H}+\mathrm{M}$ & & $\mathrm{N}$ \\
\hline $\begin{array}{l}\text { Lower } 90 \% \text { CI limit for } \\
\text { upper limit of reference } \\
\text { range }\end{array}$ & H-M & & $\mathrm{P}$ \\
\hline $\begin{array}{l}\text { Upper } 90 \% \text { CI limit for } \\
\text { lower limit of reference } \\
\text { range }\end{array}$ & $\mathrm{J}+\mathrm{M}$ & & Q \\
\hline $\begin{array}{l}\text { Lower } 90 \% \text { CI limit for } \\
\text { lower limit of reference } \\
\text { range }\end{array}$ & $\mathrm{J}-\mathrm{M}$ & & $\mathrm{R}$ \\
\hline $\begin{array}{l}90 \% \mathrm{CI} \text { of the mean } \\
\text { value }\end{array}$ & $\begin{array}{l}\text { CONFIDENCE.T(1- } \\
\mathbf{0 . 9}, \mathrm{B}, \mathrm{C})\end{array}$ & $\begin{array}{l}\text { Using t distribution. } \\
\text { Typically } 90 \% \mathrm{CI} \text { is used }\end{array}$ & $\mathrm{S}$ \\
\hline $\begin{array}{l}\text { Mean upper 90\% CI } \\
\text { limit }\end{array}$ & $\mathrm{A}+\mathrm{S}$ & & $\mathrm{T}$ \\
\hline $\begin{array}{l}\text { Mean lower 90\% CI } \\
\text { limit }\end{array}$ & A-S & & $\mathrm{U}$ \\
\hline
\end{tabular}




\begin{tabular}{lllll}
\hline & $\begin{array}{l}\text { Reference } \\
\text { range (ms) }\end{array}$ & $\begin{array}{l}\text { Extremes of 90\% } \\
\text { CIs of reference } \\
\text { range limits (ms) }\end{array}$ & $\begin{array}{l}\mathbf{9 0 \%} \text { CIs of reference } \\
\text { range limits as } \\
\text { proportion of the } \\
\text { reference range }\end{array}$ & $\begin{array}{l}\text { n (excl. } \\
\text { outliers) }\end{array}$ \\
\hline Site 1, 3.0T & $\mathbf{1 1 2 9}$ to $\mathbf{1 3 0 1}$ & 1102 to 1328 & $32 \%$ & 21 \\
\hline Site 2, 3.0T & $\mathbf{1 1 6 0}$ to $\mathbf{1 3 0 9}$ & 1145 to 1323 & $19 \%$ & 59 \\
\hline Site 2, 1.5T male & $\mathbf{9 3 3}$ to $\mathbf{1 0 2 0}$ & 927 to 1025 & $13 \%$ & 130 \\
\hline Site 2, 1.5T female & $\mathbf{9 6 5}$ to $\mathbf{1 0 5 4}$ & 957 to 1061 & $18 \%$ & 68 \\
\hline
\end{tabular}

Table 2. Comparison of reference ranges. 


\section{Figure/Table legends}

Figure 1. Illustrative relationship between various calculated values.

Figure 2. 3.0T data from both site 1 and site 2. Both data sets include males and females.

Center lines show the medians; box limits indicate the 25 th and 75 th percentiles as determined by R software; whiskers extend 1.5 times the interquartile range from the 25 th and 75th percentiles; no outliers are present; cross represents sample mean; data points are plotted as open circles. $\mathrm{n}=21$ (site 1$)$ and $\mathrm{n}=59$ (site 2 ) sample points.

Figure 3. $1.5 \mathrm{~T}$ data from site 2 only. Center lines show the medians; box limits indicate the 25th and 75th percentiles as determined by R software; whiskers extend 1.5 times the interquartile range from the 25 th and 75 th percentiles; cross represents sample mean; data points are plotted as open circles. Male $n=132$ (two outliers are seen) and female $n=68$ sample points.

Table 1. Series of spreadsheet calculations required for creating a reference interval and describing it. Commands are for Microsoft Excel 2010 or later but other tools have similar commands. For example if using Google Sheets change only "STDEV.S" to "STDEV", and “CONFIDENCE.T(1-0.9,B,C)” to "T.INV.2T(1-0.9,D)*B/SQRT(C)”.

Table 2. Comparison of reference ranges. 


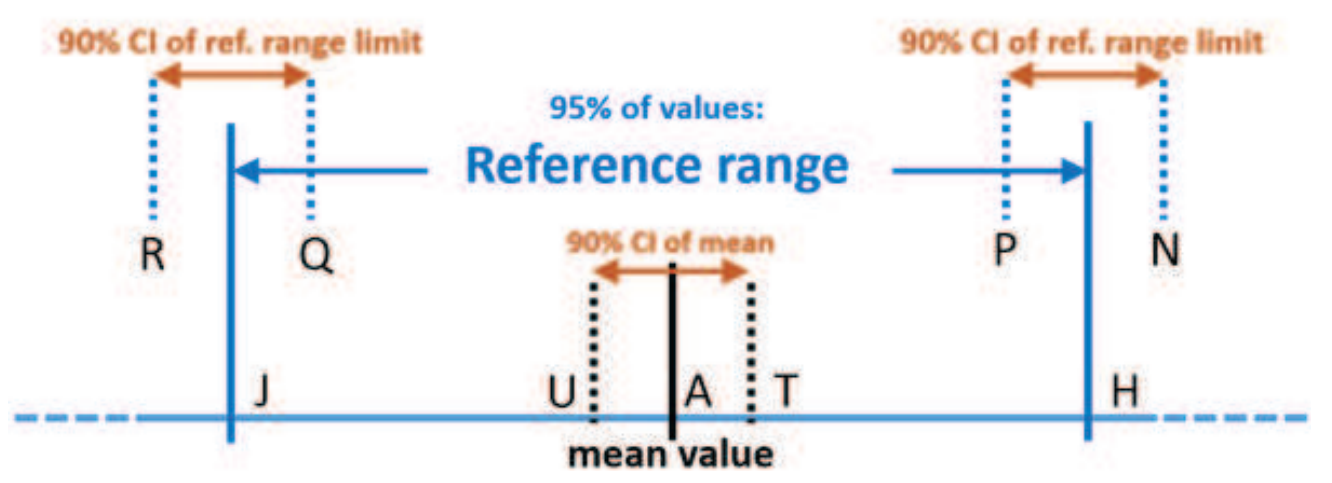

Figure 1: Illustrative relationship between various calculated values.

$51 \times 19 \mathrm{~mm}(300 \times 300$ DPI $)$ 

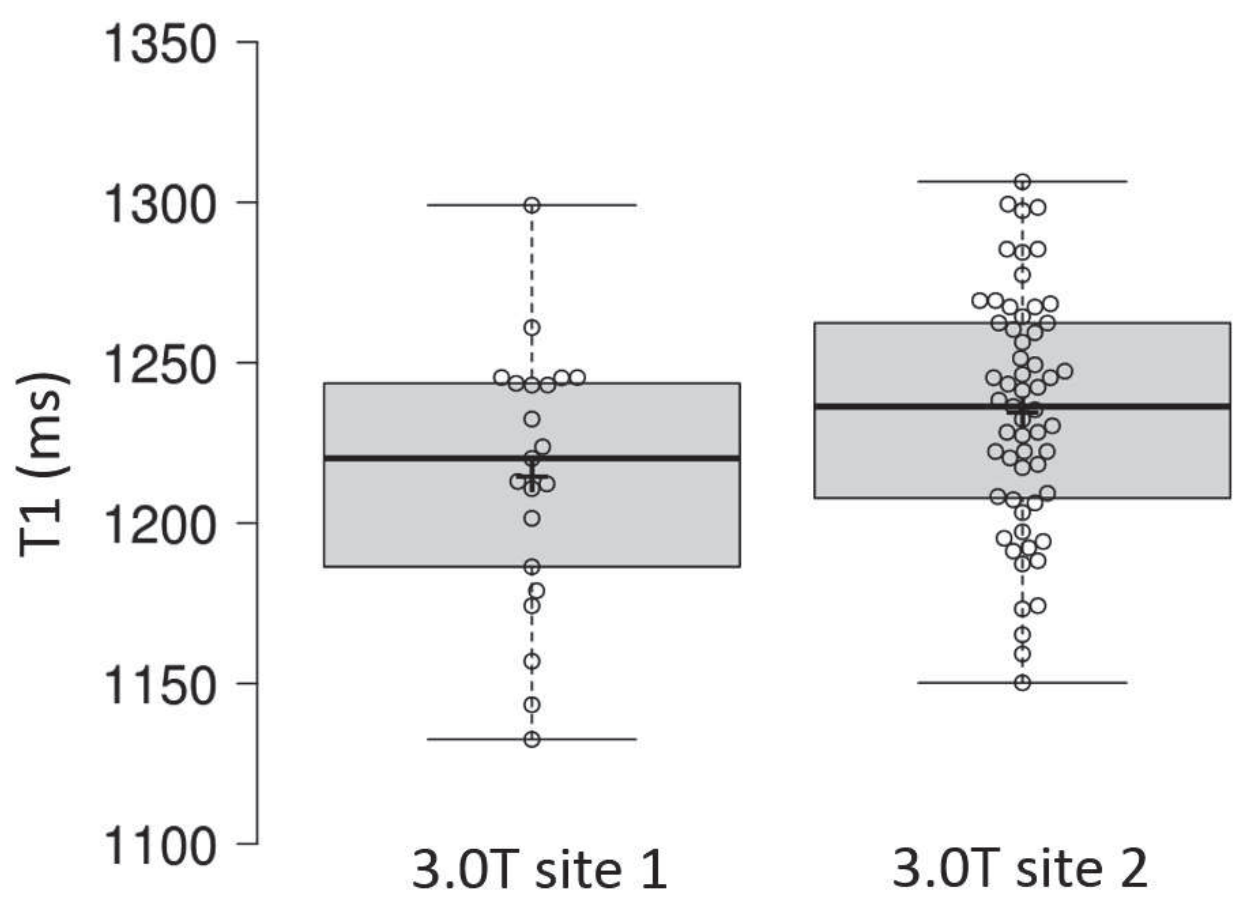

Figure 2. 3.0T data from both site 1 and site 2. Both data sets include males and females. Center lines show the medians; box limits indicate the 25th and 75th percentiles as determined by R software; whiskers extend 1.5 times the interquartile range from the 25th and 75th percentiles; no outliers are present; cross represents sample mean; data points are plotted as open circles. $n=21$ (site 1 ) and $n=59$ (site 2 ) sample points.

\section{$81 \times 57 \mathrm{~mm}(300 \times 300$ DPI $)$}




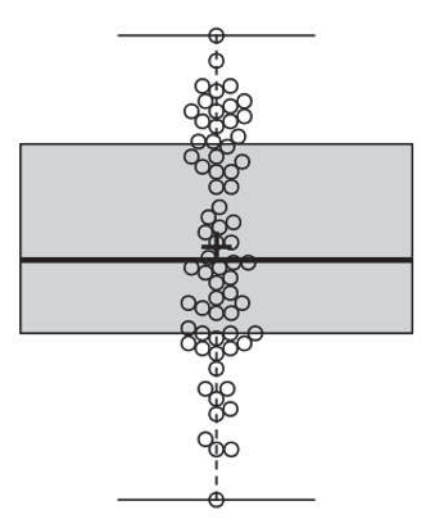

\subsection{T female}

Figure 3. 1.5T data from site 2 only. Center lines show the medians; box limits indicate the 25th and 75th percentiles as determined by $\mathrm{R}$ software; whiskers extend 1.5 times the interquartile range from the 25 th and 75th percentiles; cross represents sample mean; data points are plotted as open circles. Male $\mathrm{n}=132$ (two outliers are seen) and female $n=68$ sample points.

$85 \times 59 \mathrm{~mm}(300 \times 300 \mathrm{DPI})$ 